\title{
Nanoscale Visualization of Magnetic Contrasts with Soft X-ray Spectro- Ptychography at the Advanced Light Source.
}

$\underline{\text { Young-Sang Yu }}^{1^{*}}$, Richard Celestre ${ }^{1}$, Bjoern Enders ${ }^{1}$, Kasra Nowrouzi ${ }^{1}$, Howard Padmore ${ }^{1}$, Tony

Warwick $^{1}$, Jong-Ryul Jeong ${ }^{2 *}$ and David A. Shapiro ${ }^{1 *}$

1. Advanced Light Source, Lawrence Berkeley National Lab, Berkeley, CA 94720, USA

2. Department of Materials Science and Engineering, Graduate school of Energy Science and Technology, Chungnam National University, Daejeon 305-764, Rep. of Korea.

* Corresponding author, email: ysyu@lbl.gov; jrjeong@cnu.ac.kr; dashapiro@lbl.gov

The demand for magnetic imaging tools for understanding complex spin textures at relevant length scales (sub-10 nm spatial resolution) is rising because nanoscale correlations between magnetic and morphological heterogeneities play a key role in non-collinear spin configurations, which have been widely considered as data carriers in spintronic devices [1]. Lorentz transmission electron microscopy (LTEM) combined with aberration-correction still offers the highest spatial resolution [2], but imposes challenges involving sample thickness and retrieval of spin configurations from the phase shifts of electrons (i.e. measurable quantities in LTEM). A complementary choice is synchrotron-based soft X-ray microscopy coupled with circular-polarization, which induces a magnetic circular dichroism (MCD) according to relative orientations between the incident X-ray and magnetizations of the magnetic system. The limitation in spatial resolution of conventional scanning (STXM) and full field (FF TXM) X-ray microscopes has recently been overcome by soft X-ray ptychographic microscopy, a diffractive imaging method with a demonstrated spatial resolution better than $5 \mathrm{~nm}$ and $11 \mathrm{~nm}$ in two- and three-dimensions, respectively [3-6].

Here, we report the capabilities of soft X-ray ptychography at beamline 7.0.1.2 at the Advanced Light Source to visualize spin configurations of Fe/Gd multilayer with curvilinear defects that stabilize noncollinear spin textures in maze-like magnetic domains with perpendicular magnetic anisotropy (PMA). We adopt polarization dependent ptychography with dichroic scattering contrast near $\mathrm{Fe}$ and $\mathrm{Gd}_{\mathrm{L}} \mathrm{L}_{3}$ absorption edges to produce element-specific MCD contrast images to distinguish magnetic and structural textures. The structural and magnetic spatial resolutions of $\sim 10 \mathrm{~nm}$ were confirmed by a Fourier Ring Correlation (FRC) analysis. Figure 1 shows optical density maps collected by a conventional STXM with $25 \mathrm{~nm}$ step and reconstructed ptychographic images with $5 \mathrm{~nm}$ pixels. All images were measured at 708 $\mathrm{eV}$ with left- and right-circular polarized X-ray. As the MCD changes its sign with opposite polarizations of the incident X-ray (Fig. 1), the magnetic and mass contrasts are distinguished (Fig. 2). Due to the high spatial resolution in structural and magnetic contrasts, the detailed spin configuration near the domain walls and peculiarities induced by geometrically curved magnetic objects were revealed. Theoretical works have predicted that the existence of a curvature-induced anisotropy can stabilize topologically nontrivial radial configurations, such as circular or bubble domains and skyrmion [7].

In this demonstration of high resolution X-ray magnetic imaging, we employ circular-dichroic ptychography as an imaging tool that is applicable to a wide variety of materials and sample geometries. Although, the spatial resolution of magnetic contrasts is still far from the scale of magnetic exchange length, the coherent flux expected to increase by two to three orders of magnitude in the upgraded Advanced Light Source (ALS-U) will bring soft X-ray magnetic ptychography down to a spatial resolution of few nanometers. In addition, increasing coherent flux enables fast image acquisitions, which is essential 
to tomography, and thus allow us to investigate full three-dimensional vector imaging of magnetic systems.

[1] R. Wiesendanger, Nat. Rev. Mater. 1 (2016) 16044.

[2] S. McVitie and M. Cushley, Ultramicroscopy 106 (2006) 423.

[3] D. A. Shapiro et al., Nat. Photonics 8 (2014) 765.

[4] Y-.S. Yu et al., Nano. Lett. 15 (2015) 4282.

[5] Y-.S. Yu et al., Nat. Commun. 9 (2018) 921.

[6] X. Shi et al., Appl. Phys. Lett. 108 (2016) 094103.

[7] V. P. Kravchuk et al., Phys. Rev. Lett. 120 (2018), 067201.
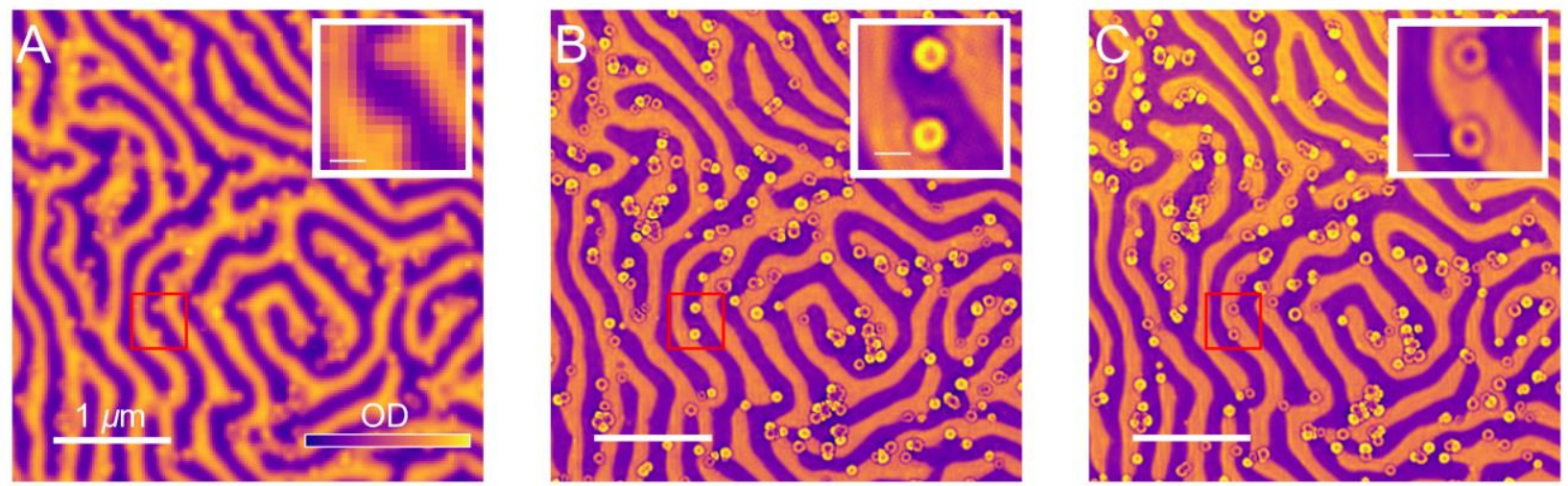

Figure 1. Magnetic transmission images of $[\mathrm{Fe}(0.543 \mathrm{~nm}) / \mathrm{Gd}(0.365 \mathrm{~nm})]_{100}$ multilayer with curvilinear defects induced by $60 \mathrm{~nm}$ polystyrene beads. Images were taken at $708 \mathrm{eV}$ by STXM with left-circularpolarization (A), ptychography with left-circular-polarization $(B)$, and ptychography with right-circularpolarization $(\mathbf{C})$. Insets show zoomed images of the identical sample position from $\mathbf{A}, \mathbf{B}$ and $\mathbf{C}$. The positions of the insets are indicated by the red boxes. All scale bars in the insets, $100 \mathrm{~nm}$.
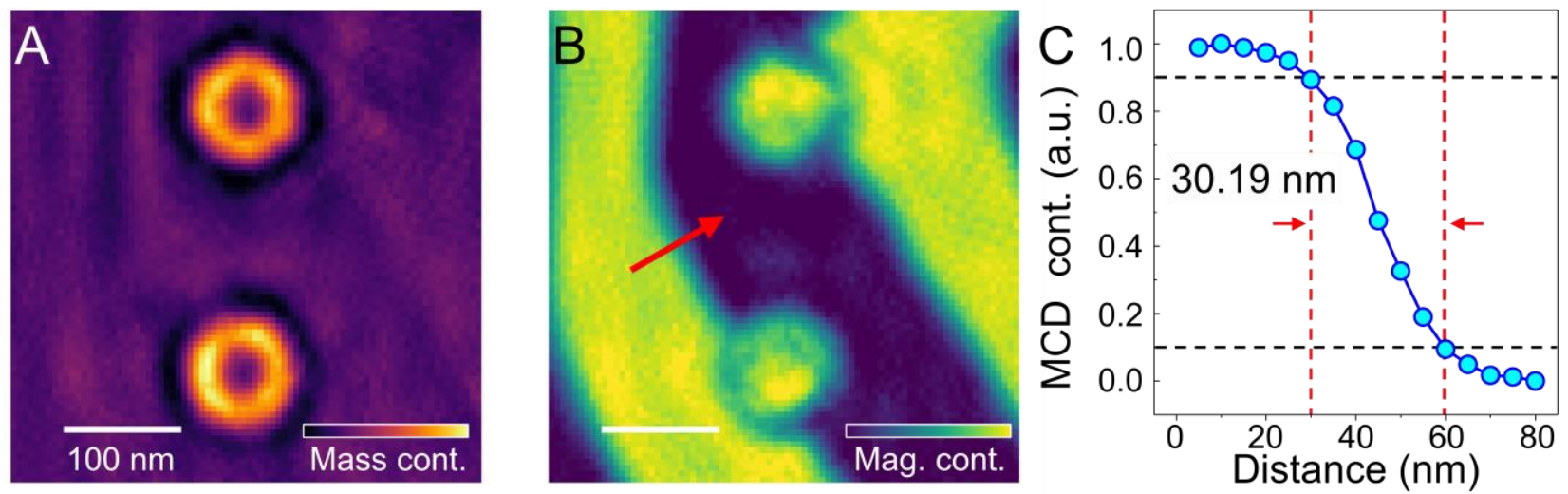

Figure 2. Mass (A) and MCD (B) contrasts near curvilinear defects calculated from ptychography images with left- and right-circular polarizations at $708 \mathrm{eV}$. The positions of the field-of-views are marked in Figs 1B and 1C. (C) Line profile of the MCD contrast indicated by the red arrow in B. Black-dashed lines are guides for $10-90 \%$ criteria. 